\title{
The use of whole-body MRI in multiple myeloma
}

Editor - We read with interest the CME masterclass on multiple myeloma. ${ }^{1}$ We would like to highlight, however, recent changes to imaging in myeloma involving whole body magnetic resonance imaging (MRI), reflected in the 2016 National Institute for Care and Health Excellence (NICE) guidance for diagnosis and treatment of this disease. ${ }^{2}$

Conventional radiographic surveys have a low sensitivity for the detection of myeloma, and are reliant on the destruction of mineralised bone, which is a relatively late finding in the disease process. Whole body MRI, a relatively recent imaging technique, has been shown to have a far higher sensitivity for the detection of bone marrow infiltration, with visualisation, before mineralised bone has been destroyed. ${ }^{3}$ The current NICE guidance recommends that MRI is used as the first line imaging in patients with suspected myeloma, for the baseline assessment of disease load in newly diagnosed patients, and for the evaluation of treatment response and suspected relapse in patients with established disease. ${ }^{2,18}$ F-labeled fluoro-2-deoxyglucose and computed tomography (CT) is useful for the detection of extra-osseous lesions, with conventional $\mathrm{CT}$ recommended in patients in whom MRI is unsuitable. Skeletal surveys are reserved only for those patients where neither MRI nor $\mathrm{CT}$ is appropriate. Conventional bone scans have no role in the current diagnostic pathway.

There is also increasing evidence of the efficacy of wholebody MRI in the assessment of patients with monoclonal gammopathy of undetermined significance and also smouldering myeloma. The diagnostic value of whole-body MRI in myeloma is enhanced by the use of functional MR techniques, namely diffusion weighted imaging (DWI) and dynamic contrast enhancement (DCE). ${ }^{4}$

Whole-body DWI is sensitive to changes in the stochastic Brownian motion and diffusion of water molecules. Myelomatous infiltration is characterised by restriction in diffusion when compared with normal marrow, thought to be due to the absence of fat cells, higher cellularity and the destruction of trabecular bone. ${ }^{3}$ These lesions appear as focal areas of increased diffusivity compared to the normal background marrow (Fig 1). DCE utilises myeloma induced angiogenesis to detect and monitor changes in bone marrow microcirculation. ${ }^{3}$

The use of whole-body MRI, including the functional techniques of DCE and DWI, has provided valuable insights into the assessment of bone marrow in patients with plasma cell dyscrasias. There are, however, some limitations on how this will be achieved in practice. MRI is relatively costly, is not always appropriate in very sick patients and may be unsuitable if, for example, the patient suffers with claustrophobia or has incompatible metallic implants. There are also resourcing and reporting issues to consider. Nevertheless, wider uptake of wholebody MRI has the potential to translate into improved patient outcomes through earlier diagnosis, improved surveillance and better characterisation of response to treatment.

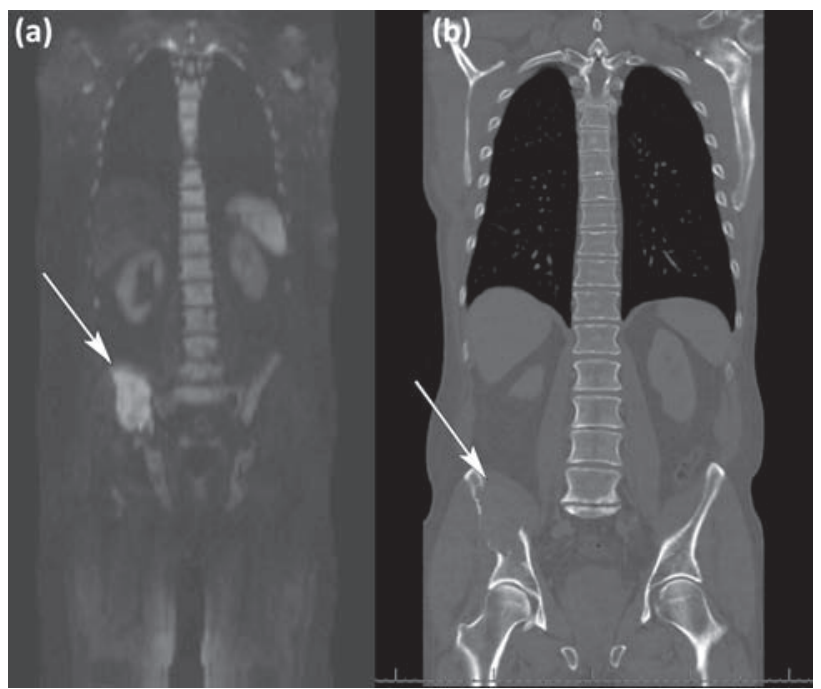

Fig 1. (a) Coronal diffusion weighted imaging showing hyperintense lesion, in the right pelvis which is restricting diffusion, as well as restriction in the vertebrae and ribs, in keeping with diffuse myeloma involvement. (b) Corresponding coronal computed tomography on bone windows, demonstrating gross destruction of the right pelvis, with an associated soft tissue lesion; the rib and vertebral lesions are less conspicuous on computed tomography and, if subtle, may not be seen. It is important to note that while sites of predilection for disease include the axial skeleton, spine and pelvis, the extra axial skeleton such as the ribs - as in this case - shoulders, skull and proximal femur are also often involved, and this justifies the use of whole-body imaging.

MARK PORTET

Radiology registrar, Eastbourne District General Hospital,

Eastbourne, UK

EMMA OWENS

Consultant radiologist, Eastbourne District General Hospital, Eastbourne, UK

DAVID HOWLETT

Consultant radiologist, Eastbourne District General Hospital, Eastbourne, UK

\section{References}

1 Firth ] (ed). Haematology: multiple myeloma. Clin Med 2019;19:58-60.

2 National Institute for Care and Health Excellence. Myeloma: diagnosis and management. NICE guideline [NG35]. NICE, 2016. www.nice.org. uk/guidance/ng35/evidence/full-guideline-pdf-2306487277.

3 Dutoit JC, Verstraete KL. MRI in multiple myeloma: a pictorial review of diagnostic and post-treatment findings. Insights Imaging 2016:7:553-69. 
4 Dutoit JC, Vanderkerken MA, Anthonissen J, Dochy F, Verstraete KL. The diagnostic value of SE MRI and DWI of the spine in patients with monoclonal gammopathy of undetermined significance, smouldering myeloma and multiple myeloma. Eur Radiol 2014;24:2754-65.
Address for correspondence: Dr Mark Portet, Eastbourne District General Hospital, Radiology Department, Kings drive, Eastbourne BN21 2UD, UK.

Email: markportet@doctors.org.uk

\section{RCP journals now available in PubMed Central}

Both ClinMed and Future Healthcare Journal are now available in PubMed Central, a web-based, free full-text archive of journal literature for all biomedical and life sciences.

Inclusion of the content back to the very first issue of both journals provides readers with greater access to valuable scholarly content and makes finding articles easier. It also means that journal content is now part of a permanent and freely accessible archive, managed by the National Library of Medicine.

FHJ can be accessed at: www.ncbi.nlm.nih.gov/pmc/journals/3619 Clin Med at: www.ncbi.nlm.nih.gov/pmc/journals/2945
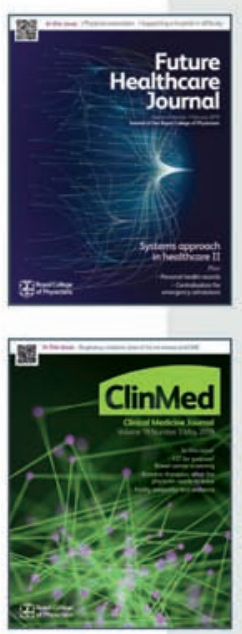